\title{
Revealing the Instantaneous Machining Gap Produced During wire Electrochemical Micromachining Using Electrochemical Deposition
}

\author{
Xiaolei Bi ${ }^{1}$, Yongbin Zeng ${ }^{1,2}$ * \\ ${ }^{1}$ Nanjing University of Aeronautics and Astronautics, Nanjing 210016, China \\ 2 Jiangsu Key Laboratory of Precision and Micro-Manufacturing Technology, Nanjing 210016, China \\ *E-mail: binyz@nuaa.edu.cn
}

doi: $10.20964 / 2020.11 .20$

Received: 3 July 2020 / Accepted: 21 August 2020 / Published: 30 September 2020

\begin{abstract}
Wire electrochemical micromachining (WECMM) has increasingly been recognized as a promising method in producing micro-components. Research into the machining gap is of great importance for understanding the regularities of WECMM. However, studies published on the machining gap are usually approximate and simplified because it is difficult to observe and measure the formation and distribution of the instantaneous machining gap. In this paper, electrochemical deposition is proposed as an innovative method for revealing the instantaneous machining gap produced during WECMM. The method is shown to be feasible in principle by a simulation. Optimum values of $1.0 \mathrm{~A} / \mathrm{dm}^{2}$ for the current density and $3 \mathrm{~h}$ for the machining time are determined experimentally. Several techniques for obtaining a clear contour of the machining gap are tested, and focused ion beam milling is finally selected as the best way to remove the redundant wire and metal deposited beyond the workpiece surface in the position of the machining gap. Using the proposed method, the contour of the machining gap during WECMM of pure nickel is clearly revealed for the first time.
\end{abstract}

Keywords: Wire electrochemical micromachining (WECMM), Machining gap, Electrochemical deposition, Focused ion beam milling (sputtering and etching)

\section{$\underline{\text { FULL TEXT }}$}

(C) 2020 The Authors. Published by ESG (www.electrochemsci.org). This article is an open access article distributed under the terms and conditions of the Creative Commons Attribution license (http://creativecommons.org/licenses/by/4.0/). 